\title{
A MAPLE Package for Integro-Differential Operators and Boundary Problems
}

\author{
Anja Korporal \\ Research Institute for Symbolic Computation, Johannes Kepler Universität Linz \\ Altenberger Str. 69, 4040 Linz, Austria \\ Anja.Korporal@risc.jku.at \\ Georg Regensburger \\ Johann Radon Institute for Computational and Applied Mathematics, \\ Austrian Academy of Sciences, Altenberger Str. 69, 4040 Linz, Austria \\ Georg . Regensburger@oeaw.ac . at \\ Markus Rosenkranz \\ University of Kent, Cornwallis Building, Canterbury, Kent CT27NF, United Kingdom \\ M. Rosenkranz@kent .ac.uk
}

Introduction. We present a MAPLE package for computing in algebras of integro-differential operators. This provides the appropriate algebraic setting for treating boundary problems [7] for linear ordinary differential equations symbolically. They allow to formulate a boundary problem - a differential equation and boundary conditionsbut they are also expressive enough for describing its solution via an integral operator, which is called Green's operator. The implementation was tested in MAPLE 11, 12 and 13. It is available with an example worksheet at http://www.risc.jku.at/people/akorpora/index.html.

Integro-differential Operators. The definition of integro-differential operators is based on an integro-differential algebra $\left(\mathcal{F}, \partial, \int\right)$ providing the coefficients. It is given by a commutative differential algebra $(\mathcal{F}, \partial)$ over a field $K$ such that $\int$ is a $K$-linear right inverse of $\partial$ that fulfills the differential Baxter axiom

$$
\left(\int f^{\prime}\right)\left(\int g^{\prime}\right)=\left(\int f^{\prime}\right) g+f\left(\int g^{\prime}\right)-\int(f g)^{\prime}
$$

see [5] for details. For the similar notion of differential Rota-Baxter algebras see [1]. We call an integro-differential algebra ordinary if $\operatorname{dim}_{K} \operatorname{ker}(\partial)=1$. The integro-differential operators $\mathcal{F}_{\Phi}\left[\partial, \int\right]$ over an ordinary integro-differential algebra $\left(\mathcal{F}, \partial, \int\right)$ are introduced in [5] essentially as the $K$-algebra generated by the symbols $\partial$ and $\int$, the functions $f \in \mathcal{F}$ and a collection of multiplicative functionals $\Phi \subseteq \mathcal{F}^{*}$, modulo a Noetherian and confluent rewrite system. In our MAPLE package, we do not implement the rewrite rules but a new approach via normal forms. The key fact for working with normal forms is that each integro-differential operator can be written uniquely as a sum of a differential, an integral, and a so-called Stieltjes boundary operator. Furthermore, the normal forms of products within and between these three classes of operators are straightforward to determine [3]. Differential operators are well-known, normal forms of integral operators are sums of terms of the form $f \int g$ with $f, g \in \mathcal{F}$ and boundary operators are sums of terms having the form $f \varphi \partial^{i}$ or $f \varphi \int g$ with $\varphi \in \Phi$ and $f, g \in \mathcal{F}$. In our implementation, the functionals in $\Phi$ are arbitrary point evaluations. As "coefficient algebra" we use the fragment of $\mathcal{C}^{\infty}$ functions representable in MAPLE. The differential operator $\partial$ is the usual derivation and the integral operator $\int$ is the integral $\int_{0}^{x}$, both computed by MAPLE.

Boundary problems. The idea of solving boundary problems symbolically was presented in [4] for two-point boundary problems with constant coefficients and generalized to the setting of integro-differential algebras in [5]. 
A general boundary problem of order $n$ is formulated as follows: Given a forcing function $f \in \mathcal{F}$, we want to find $u \in \mathcal{F}$ such that

$$
\begin{aligned}
& T u=f \\
& \beta_{1} u=\cdots=\beta_{n} u=0
\end{aligned}
$$

for a monic differential operator $T$ of order $n$ and Stieltjes boundary conditions $\beta_{1}, \ldots, \beta_{n} \in \mathcal{F}^{*}$. For regular boundary problems, the Green's operator $G: \mathcal{F} \rightarrow \mathcal{F}$ maps the forcing function $f$ to the unique solution $u$. Our package computes the Green's operator for a given regular boundary problem, provided that MAPLE can solve the underlying homogeneous differential equation $T u=0$. For computing its fundamental system, we use the MAPLE package DEtools and the command dsolve. An interesting example is given by the differential equation

$$
u^{\prime \prime \prime}-\left(e^{x}+2\right) u^{\prime \prime}-u^{\prime}+\left(e^{x}+2\right) u=f,
$$

for which MAPLE can find the general solution and solve it with given initial conditions. But it seems to have no systematic procedure to solve it for arbitrary boundary conditions. For example, MAPLE does not give any solution for the conditions

$$
u(0)=u(1)=u^{\prime}(1)+u^{\prime \prime}(0)=0 .
$$

With our package, we can compute the Green's operator for this boundary problem and apply it symbolically to an arbitrary forcing function $f$. The output is part of our example worksheet.

Outlook. We plan to have a complete package for treating linear boundary problems for ordinary differential equations. We have already implemented various operations on boundary problems, like composition and factorization of boundary problems along a given factorization of the defining differential operator $[5,6]$, see also the example worksheet. In a next step, we want to treat singular boundary problems, as for example in [2]. The package should also allow for an extension to integro-differential operators acting on multivariate functions; see [6] for some first steps in this direction.

\section{References}

[1] L. Guo and W. Keigher. On differential Rota-Baxter algebras. J. Pure Appl. Algebra, 212(3):522-540, 2008.

[2] W. S. Loud. Some examples of generalized Green's functions and generalized Green's matrices. SIAM Rev., 12:194-210, 1970.

[3] G. Regensburger, M. Rosenkranz, and J. Middeke. A skew polynomial approach to integro-differential operators. In John P. May, editor, Proceedings of ISSAC '09, pages 287-294. ACM Press, 2009.

[4] M. Rosenkranz. A new symbolic method for solving linear two-point boundary value problems on the level of operators. J. Symbolic Comput., 39(2):171-199, 2005.

[5] M. Rosenkranz and G. Regensburger. Solving and factoring boundary problems for linear ordinary differential equations in differential algebras. J. Symbolic Comput., 43(8):515-544, 2008.

[6] M. Rosenkranz, G. Regensburger, L. Tec, and B. Buchberger. A symbolic framework for operations on linear boundary problems. In Proceedings of CASC '09, volume 5743 of LNCS, pages 269-283, Berlin, 2009. Springer.

[7] I. Stakgold. Green's functions and boundary value problems. Pure and Applied Mathematics (New York). John Wiley \& Sons Inc., New York, second edition, 1998. A Wiley-Interscience Publication. 